\title{
Detection of chromosome aberrations in metaphase and interphase tumor cells by in situ hybridization using chromosome-specific library probes
}

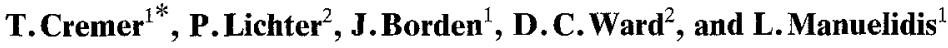 \\ ${ }^{1}$ Section of Neuropathology and ${ }^{2}$ Department of Human Genetics, Yale University School of Medicine, New Haven, CT 06510, USA
}

\begin{abstract}
Summary. Chromosome aberrations in two glioma cell lines were analyzed using biotinylated DNA library probes that specifically decorate chromosomes $1,4,7,18$ and 22 from pter to qter. Numerical changes, deletions and rearrangements of these chromosomes were readily visualized in metaphase spreads, as well as in early prophase and interphase nuclei. Complete chromosomes, deleted chromosomes and segments of translocated chromosomes were rapidly delineated in very complex karyotypes. Simultaneous hybridizations with additional subregional probes were used to further define aberrant chromosomes. Digital image analysis was used to quantitate the total complement of specific chromosomal DNAs in individual metaphase and interphase cells of each cell line. In spite of the fact that both glioma lines have been passaged in vitro for many years, an under-representation of chromosome 22 and an over-representation of chromosome 7 (specifically $7 p$ ) were observed. These observations agree with previous studies on gliomas. In addition, sequences of chromosome 4 were also found to be under-represented, especially in TC 593. These analyses indicate the power of these methods for pinpointing chromosome segments that are altered in specific types of tumors.
\end{abstract}

\section{Introduction}

Genetic changes are central to the initiation and progression of neoplasias. Some changes, such as point and insertional mutations, are submicroscopic. Other changes are quite large, and can be detected grossly in chromosome analyses. Nonrandom chromosomal changes, especially in hematopoietic malignancies, have been well established in recent years, and often mirror events at the molecular level. Such non-random changes may occur at an early stage in tumorigenesis and can be characteristic or even unique for specific tumor types (Showe and Croce 1987; Bloomfield et al. 1987; Hansen and Cavenee 1987). Non-random changes are also associated with an increased metastatic potential (Larizza and Schirrmacher 1984). Chromosomes can exhibit highly complex structural alterations, especially in some epithelial tumors, and these complex alterations are often associated with progressive biologi-

* Present address: Institut für Humangenetik und Anthropologie der Universität, Im Neuenheimer Feld 328, D-6900 Heidelberg 1, Federal Republic of Germany

Offprint requests to: L. Manuelidis cal features of malignancy that are detrimental to the organism. In this setting it is important to be able to rapidly and easily assess chromosomal segments involved in complex duplications, deletions or translocations.

Cytogenetic investigations of cells derived from human solid tumors are tedious and difficult to perform, due to both the complexity of chromosomal changes and to the lack of sufficient numbers of suitable metaphase spreads for reliable analyses. In many cases, it may be difficult or even impossible to assess chromosomal aberrations by conventional banding techniques. Even when performed by highly experienced cytogeneticists it is the rule rather than the exception that karyotypes established from solid tumors include a number of aberrant chromosomes that are impossible to assess. Consequently, the interpretation of cytogenetic findings can be ambiguous; for example it may be impossible to determine if there is a deletion of part of an identified chromosome, or if this missing segment is actually present as a translocation on another chromosome.

To overcome these limitations we have developed a new approach by which individual human chromosomes can be specifically stained at any time of the cell cycle and used to identify chromosomal aberrations, not only in mitotic cells but also in more numerous examples of interphase nuclei. Previous experiments have shown that individual chromosome segment probes are useful in this setting (Rappold et al. 1984; Burns et al. 1985; Cremer et al. 1986, 1988; Trask et al. 1988). In this paper we delineate entire single chromosomes in model glioma cells that display extremely complex chromosomal alterations. In such studies translocations were reliably identified. Our approach is based on chromosomal in situ suppression hybridization of DNA libraries from sorted human chromosomes to both human mitotic and interphase cells, and is described in detail in the accompanying paper (Lichter et al. 1988). We refer to this approach as CISS hybridization. Using two glioma lines, TC 620 and TC 593 (Manuelidis and Manuelidis 1979), we demonstrate that it is possible to rapidly screen both mitotic and interphase tumor cells for complex numerical and structural aberrations of individual chromosomes. Despite the fact that these oligodendroglioma and glioblastoma lines were propagated in long term culture, they displayed several cytogenetic features common to many glioma cells (Shapiro 1986; Bigner et al. 1986, 1987; Rey et al. 1987).

In the present study we have specifically focused on chromosomes 1, 4, 7, 18 and 22. These chromosomes were selected because (1) they span the entire range of chromosome size. 
(2) Numerical and structural aberrations of chromosome 1 have been found in many solid tumors (Atkin 1986). (3) Aberrations of chromosomes 1 and 18 have been previously noted in TC 620 and TC 593 by in situ hybridization with subregional DNA probes specific for $1 \mathrm{q} 12$ and the pericentromeric heterochromatin of chromosome 18 (Cremer et al. 1988). (4) Numerical imbalances of chromosomes 7 and 22 have been considered to be of special importance in the development of malignant gliomas (Shapiro 1986; Bigner et al. 1986, 1987; Ray et al. 1987).

\section{Materials and methods}

\section{Cells}

TC 593 is a pseudotetraploid cell line (modal chromosome number, 83) established from a human glioblastoma; it grows in a flat, spreading fashion and contains many processes. TC 620 is pseudotriploid with a modal chromosome number of 64 and was established from a human oligodendroglioma; it grows in an epithelial fashion. Both cell lines have been described in detail (Manuelidis and Manuelidis 1979). In the present experiments we utilized subclones C2B (TC 593) and C2B (TC 620 ) at approximately 180 passages after repeated subcloning from a single cell of the original tumor line cultured as previously described (Manuelidis and Manuelidis 1979). Standard hypotonic treatment and acid/methanol fixation of the cells were employed (Cremer et al. 1988).

\section{$D N A$ probes and libraries}

Phage DNA libraries from sorted human chromosomes were obtained from the American type culture collection: LA01NS01 (chromosome 1), LL04NS01 (chromosome 4), LA07NS01 (chromosome 7), LL18NS01 (chromosome 18) and LA22NS03 (chromosome 22). Amplification of these libraries, isolation of human DNA inserts and biotinylation were carried out as described by Lichter et al. (1988). A probe specific for alphoid repeats on chromosome 7 ( $\mathrm{p} \alpha \mathrm{t} \mathrm{t} 1$ ) was the gift of $\mathrm{H}$. Willard (Waye et al. 1987), and specifically decorates centromeric heterochromatin of chromosome 7 under high stringency conditions ( $60 \%$ formamide; see Cremer et al. 1988). Some DNA probes were modified with aminoacetylfluorene (AAF; Landegent et al. 1984), and detected as described (Cremer et al. 1988) for double labeling experiments.

\section{In situ hybridization and detection of hybridized probes}

CISS hybridization with biotinylated library DNA inserts and detection of hybrid molecules was generally carried out using standard conditions, as described in detail by Lichter et al. 1988. In double CISS hybridizations using biotinylated chromosome 7 library DNA inserts and the AAF-modified 7 alphoid probe, the latter probe was heat denatured separately and only added to the hybridization mixture at the end of the reannealing step (see Lichter et al. 1988) at a final concentration of $10 \mu \mathrm{g} / \mathrm{ml}$.

\section{Digital image analysis of specifically decorated metaphase and interphase chromosomes}

A VAX station II/GPX graphics workstation (Digital Equipment Corporation) with an ITEX FG 100-Q frame grabber (Imaging Technology) were used as previously described (Manuelidis and Borden 1988), together with a Zeiss S-Planar $60 \mathrm{~mm}$ lens and a Dage-MTI 65 video camera. Images were digitized from negatives of metaphase spreads and interphase nuclei; the background was removed and polygonal regions were defined to enclose specifically decorated metaphase chromosomes or interphase domains (Lichter et al. 1988). A scan line algorithm was used to calculate histograms within the polygonal regions. Since the value of the histogram $\mathrm{H}(i)$ of a particular intensity (range 0-255) within the defined region is the number of pixels at that intensity $i$, the area within the region falling within an intensity range $i_{0}-i_{1}$ is the integral of the histogram from $i_{0}-i_{1}$. Similarly, the 2-D integral in the region defined by the intensity range $i_{0}-i_{1}$ equals $\sum \mathrm{H}(i) \cdot i$. $i_{\mathrm{o}}$ was chosen for each hybridization, in order to properly outline the decorated chromatin and distinguish this area from background regions. $i_{1}$ was set to the maximum value 255 in order to capture the entire intensity range above the threshold.

Measurements of total signal intensity versus area were designed as a control for the potential presence of variable chromosome domain extension within interphase nuclei. In interphase, a more extended chromosome domain might be expected to have a greater area (or volume) yet a lower fluorescence signal intensity per unit area. If a constant amount of hybridized DNA corresponds to a constant total fluorescence, the total signal intensity is a measure of labeled DNA content. We can also measure 3-D hybridized volumes within nuclei (Manuelidis and Borden 1988) and 3-D integrated total hybridized signals (J. Borden and L. Manuelidis, in preparation). The background, $b$, was substracted from the discrete 2-D integral $\iint \mathrm{i}(\mathrm{x}, \mathrm{y}) \mathrm{dA}$ within a labeled region $\mathrm{R}$, to yield the total signal: $\operatorname{Sig}_{t}=\iint \mathrm{i}(\mathrm{x}, \mathrm{y}) \mathrm{dA}-\mathrm{b} \iint \mathrm{dA}$, where $\mathrm{dA}$ is a single pixel. Similarly, the mean intensity within the region is calculated as 2-D integral/area or $\iint \mathrm{i}(\mathrm{x}, \mathrm{y}) \mathrm{dA} / \iint \mathrm{dA}$.

\section{Results}

Detection of numerical and structural chromosome aberrations in metaphase spreads

Figures 1-3, and 7 show typical metaphase spreads from the malignant glioma cell lines TC 620 and 593 after CISS hybrid-

Fig. 1A-D. Chromosomal in situ suppression (CISS) hybridization of chromosome 1 inserts to metaphase spreads of glioma cell lines detected with avidin-FITC (A, C) and poststained with 4,6-diamidino-2-phenylindole dihydrochloride (DAPI) (B, D). A, B TC 620 shows two apparently complete 1 chromosomes (small arrows in $\mathbf{B}$ ) and two marker translocation chromosomes (arrowheads in B) specifically decorated by these inserts (A). One of the two marker chromosomes contains a 1p (lower left), the other a 1q arm (lower right); the 1p terminal (relatively GC rich region) in the two normal chromosomes and submetacentric marker is less completely delineated. Also, the $1 \mathrm{q} 12$ regions here show little decoration in contrast to most experiments. $\times 950$. C, D Typical TC 593 metaphase spread shows six specifically decorated chromosomes. Three acrocentric marker chromosomes all with truncation of $1 \mathrm{p}$ show particularly intense fluorescence of repeats that localize to $1 \mathrm{q} 12$ (arrows in $\mathbf{C}$ ). In two of these, 1q arms may be complete, while a major deletion is obvious in the third (arrows in $\mathbf{D}$ ). A fourth decorated chromosome (small arrowhead in $\mathbf{C}, \mathbf{D})$ again shows a major deletion of the distal part of $1 \mathrm{q}$, but has retained an apparently complete $1 \mathrm{p}$ arm. A fifth submetacentric chromosome (large arrowhead in $\mathbf{D}$ ) contains an apparently complete $1 \mathrm{p}$ arm; the DNA of its short arm has not been identified. Note the similarity of this marker to one of the marker chromosomes of TC $620(1 \mathrm{p})$ described above. The sixth entirely decorated chromosome is an iso(1p) as demonstrated by DAPI-banding (open arrows in D). $\times 1200$ 


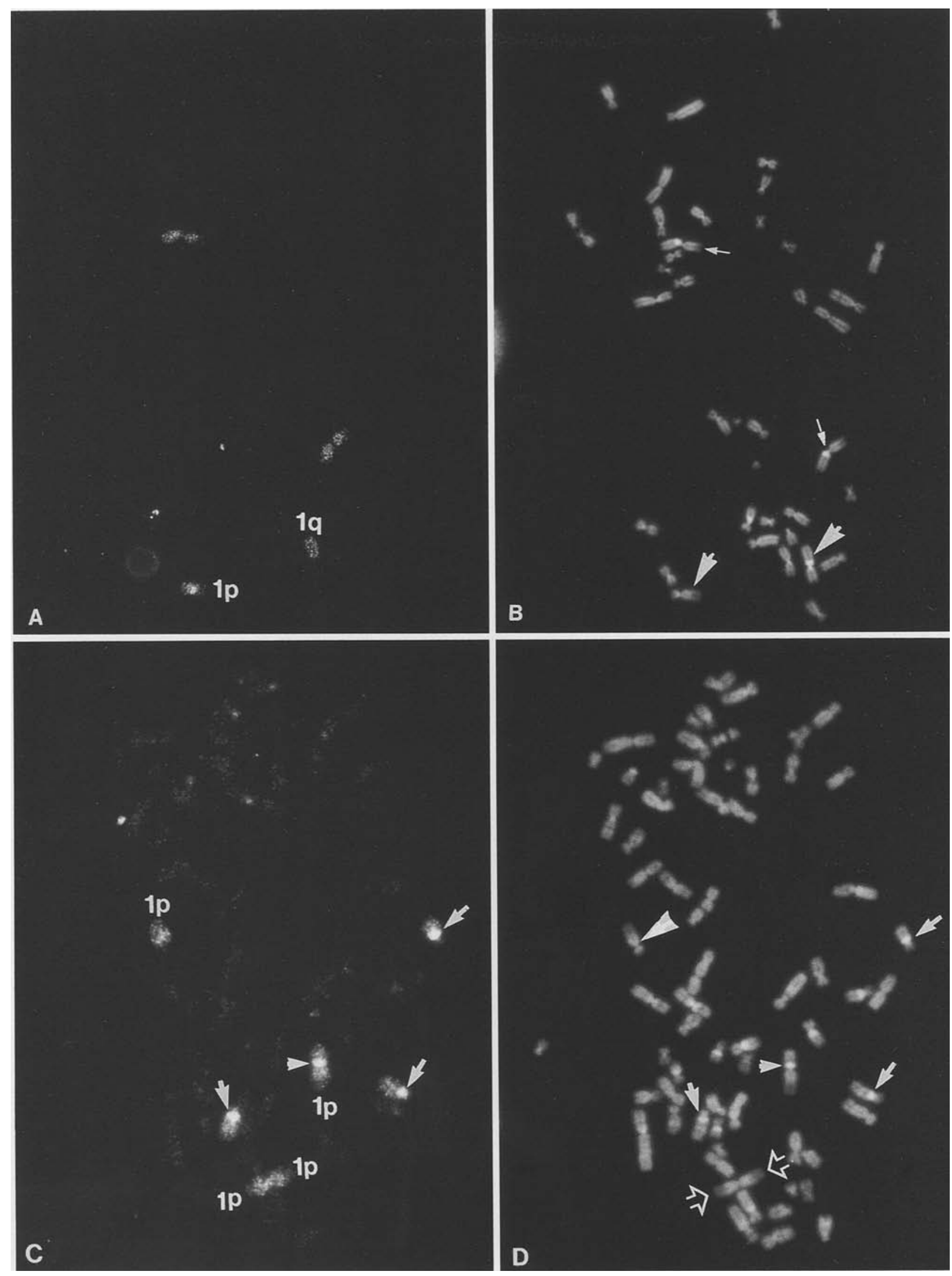



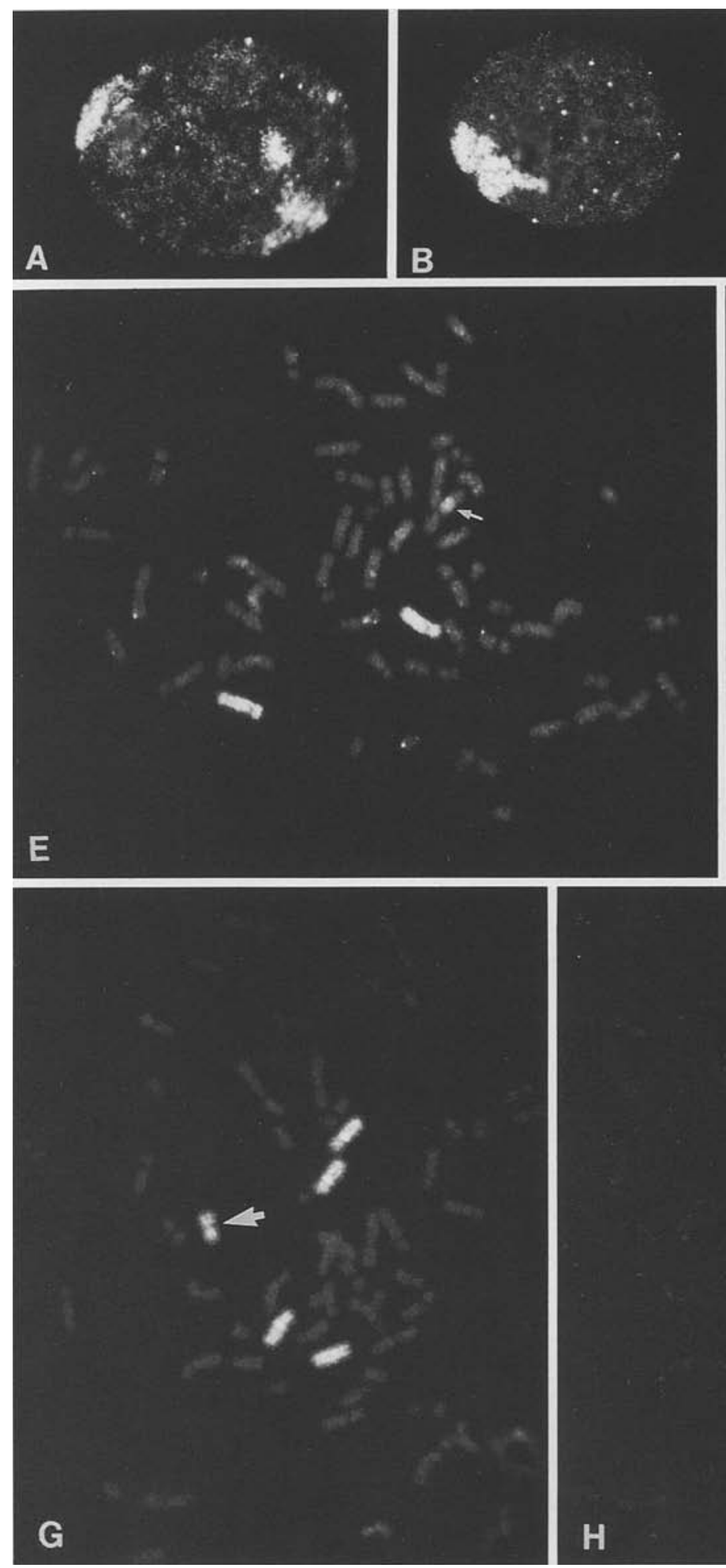
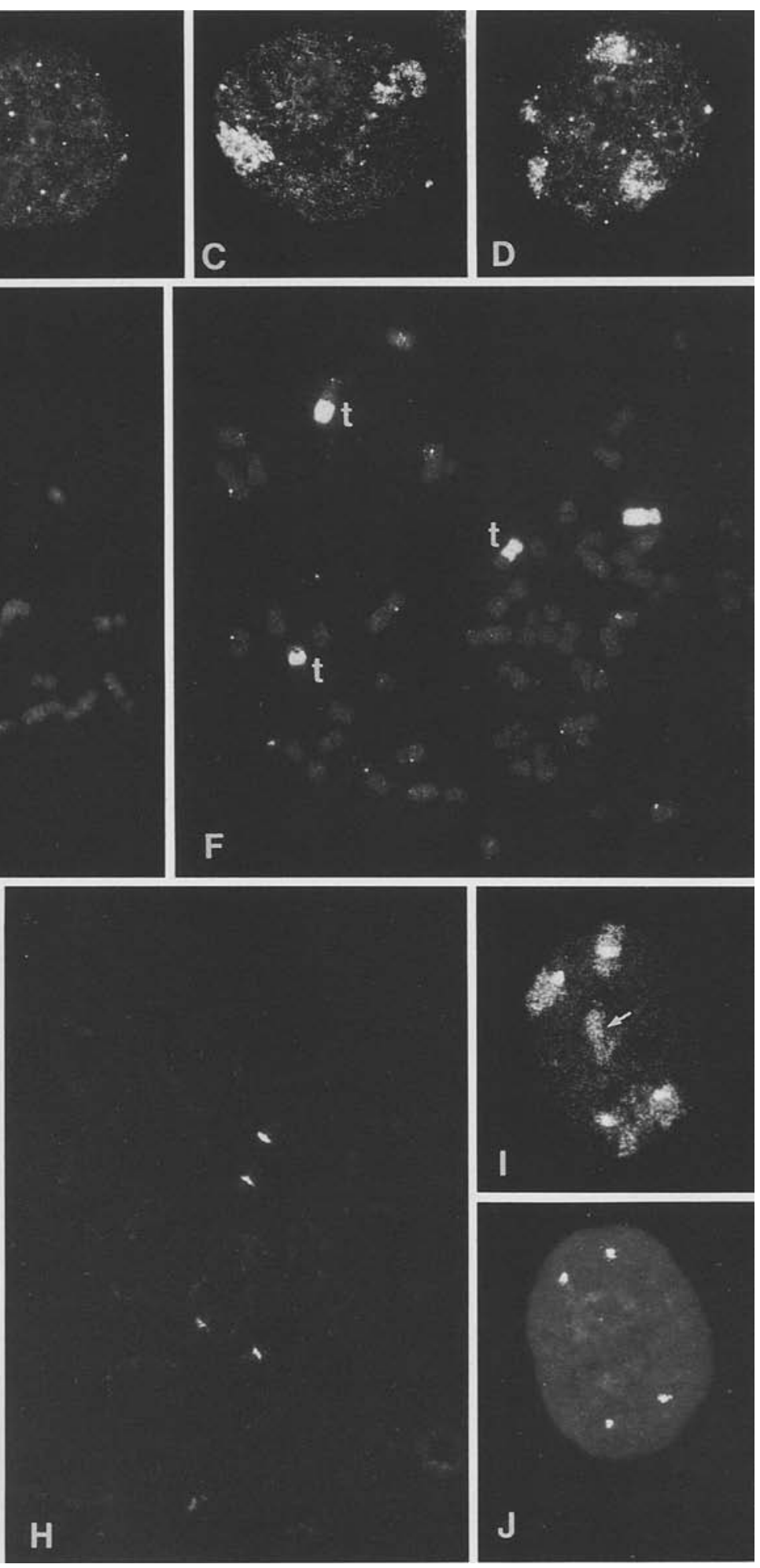

Fig. 2. A-F CISS hybridization of chromosome 4 library inserts detected with avidin-FITC; interphase nuclei of TC 593 (A-C) and TC 620 (D). Note that the two apparently complete interphase domains are close to each other in $\mathbf{B}$, but widely separated in $\mathbf{A}$ and $\mathbf{C}$. TC 620 interphase nucleus (D) shows four chromosome 4 interphase domains of largely different sizes. Metaphase spread of TC 593 (E) shows two apparently complete 4 chromosomes, and a small decorated ragion (arrow) in a submetacentric chromosome. This marker with translocated 4 sequences was observed in about $30 \%$ of the spreads and is also apparent in the interphase nucleus represented in $\mathbf{A}$. TC 620 metaphase spread (F) shows one apparently complete chromosome 4 and three translocation markers $(t)$ containing different amounts of chromosome 4 material. G-J Double hybridization of biotinylated chromosome 7 inserts and an aminoacetylfluorene (AAF)-modified 7-specific alphoid repeat. G Chromosome 7 inserts depict five entirely decorated metaphase chromosomes. Four of them are complete 7 chromosomes, the fifth (arrow) is an iso(7p) (see Fig. 3E). H The same field as G showing AAF-7 alphoid signals on only four decorated chromosomes; no signal is detected on the iso( $7 \mathrm{p})$. I An interphase nucleus of TC 593 shows five domains delineated by chromosome 7 inserts; four of these are labeled by 7 alphoid probes $(\mathbf{J})$. The arrow in $\mathbf{I}$ indicates the iso(7p) marker in interphase 


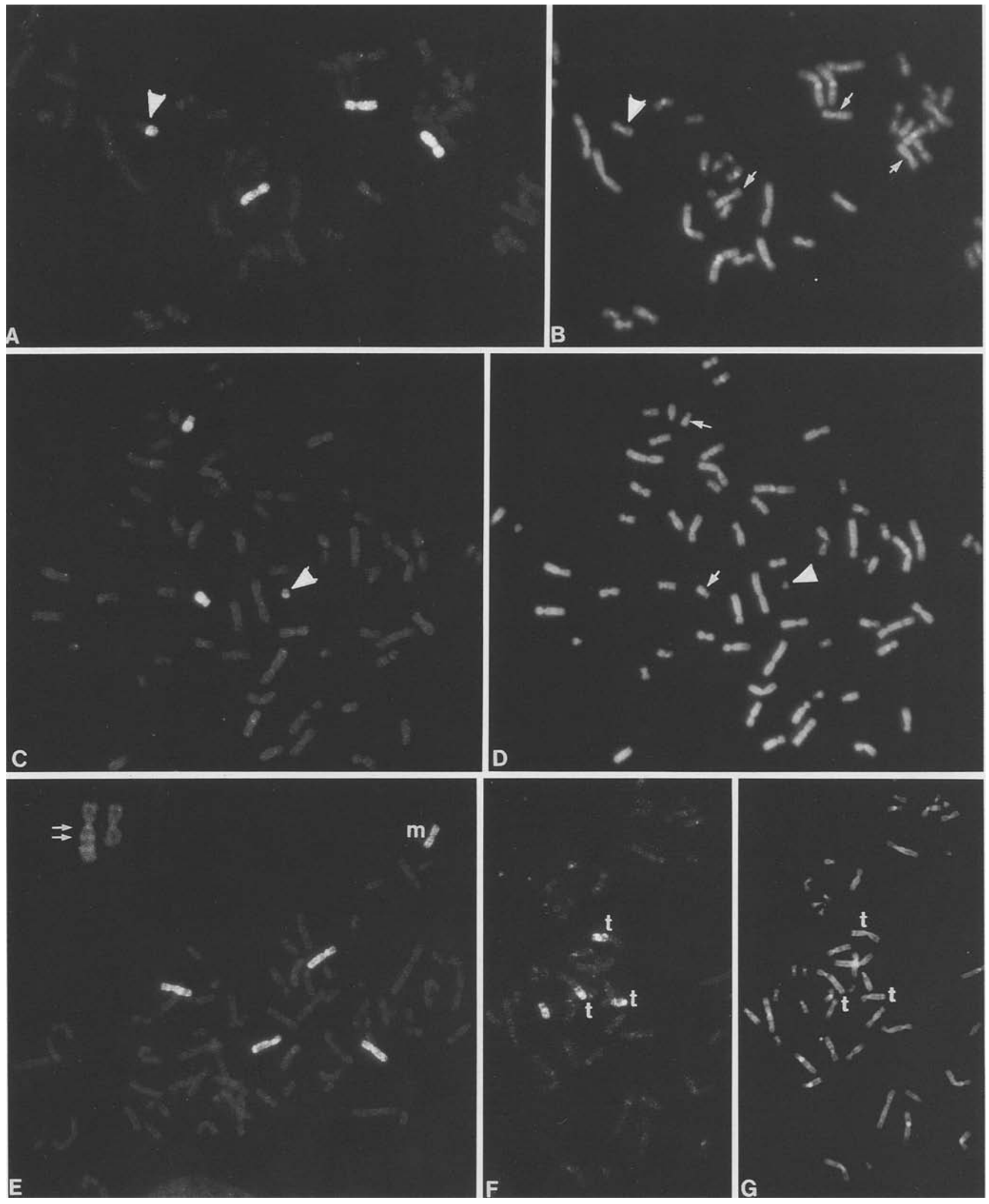

Fig. 3. A-G CISS hybridization of library inserts of chromosomes 7 and 18 to metaphase spreads of glioma cell lines detected with avidin-FITC. $\times 875$. A, B TC 620 hybridized to chromosome 7 inserts. Three apparently normal 7 chromosomes and an additional translocation chromosome containing 7 sequences (large arrowhead) are delineated in $\mathbf{A} ;$ DAPI-stained complete chromosomes 7 are indicated by the small arrows in $\mathbf{B}$. Other studies (see the text) indicated a translocation of 7pter-q11 in the marker chromosome (large arrowhead). C, D TC 620 hybridized to chromosome 18 inserts. In $\mathbf{C}$ two normal 18 chromosomes are decorated (cf. small arrows in $\mathbf{D}$ ) as well as a petite chromosome (large arrowhead). D The corresponding frame counterstained with DAPI. E Metaphase spread from pseudotetraploid TC 593 shows five chromosomes highlighted by 7 library inserts. The metacentric chromosome $(\mathrm{m})$ represents the iso(7p) marker typical for this line (see also Fig. 2G). Insert chromosomes (upper left in $\mathbf{E}$ ) show a DAPI-stained normal and the metacentric 7 marker chromosome. The landmark band $7 \mathrm{q} 21$ and a block of constitutive heterochromatin at $7 \mathrm{q} 11$ are both prominent on the normal chromosome 7 , (arrows), but not present on the marker chromosome. Instead both arms of the latter show a mirror-like staining pattern with a faint distal band at 7 p21. F, G TC 593 hybridized to chromosome 18 inserts. Four decorated 18 chromosomes are shown in $\mathbf{F}$ and three of them shown in $\mathbf{G}$ by DAPI staining are clearly translocated $(t)$ 
TC 620
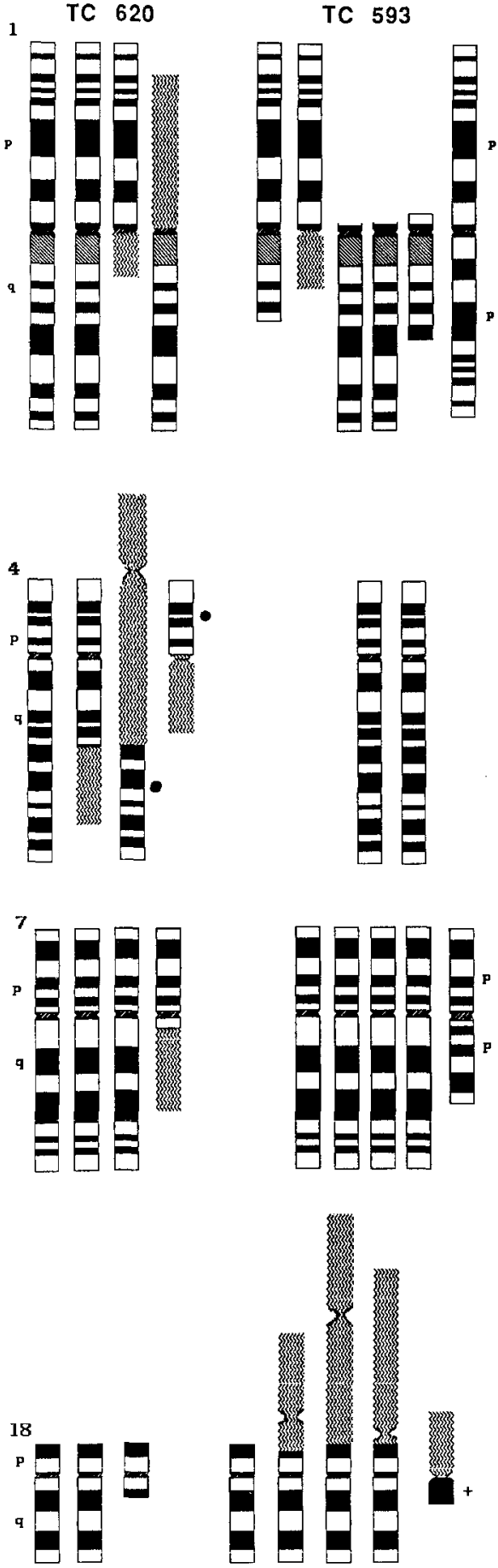

22
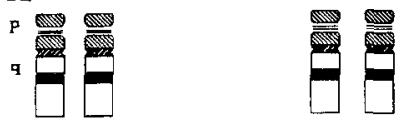

Fig. 4. Summary chromosome idiogram of complete and aberrant chromosomes detected by CISS hybridization of library inserts of chromosomes $1,4,7,18$ and 22 in glioma cell lines TC 620 (left) and TC 593 (right). G-bands (black) are shown with approximate breakpoints suggested by our data; the shaded areas with a wavy pattern are from other chromosomes that constitute part of the marker translocation chromosomes. The black dot beside two of the TC 620 translocated 4 segments indicates that the assignment of the chromosome 4 material is based on circumstantial evidence (e.g. size measurements). A small translocation of chromosome 18 material in ca. $20 \%$ of TC 593 metaphase spreads $(t)$ also could not be further identified. Note the over-representation of $7 \mathrm{p}$ in both cell lines ization with biotinylated DNA inserts from each of the human chromosomes 1, 4, 7, 18 and 22. Hybridized inserts were detected with avidin fluorescein isothiocyanate conjugates (FITC) and cells were counterstained with 4,6-diamidino-2phenylindole dihydrochloride (DAPI). Chromosomes designated as "complete" had an apparently normal size, centromere index and DAPI staining pattern. Despite this designation, these complete chromosomes may contain fine structural aberrations only detectable by additional investigations (see below). Apparently complete chromosomes 1, 4, 7, 18 and 22 were observed in both TC 620 and TC 593. Additionally, other homologs of these chromosomes showed significant rearrangements and abnormalities, including translocations and deletions. We describe below the predominant numerical and structural aberrations delineated in each of these cell lines. A minimum of 25 good metaphase spreads were evaluated for each glioma line and for each chromosome. These data are summarized in Fig. 4.

Chromosome 1. In TC 620 , the oligodendroglioma line, chromosome 1 inserts decorated two apparently complete 1 chromosomes and two marker translocation chromosomes (Figs.1A, B, 4). One marker was metacentric and contained an entirely decorated 1q arm, but its $p$ arm was from another chromosome (of unknown origin). The other marker chromosome was submetacentric and showed a small segment from another chromosome attached to the $1 p \mathrm{arm}$. In both marker chromosomes breakpoints were localized close to the centromere in $1 \mathrm{p} 11$ or $1 q 11$. The identification of the $1 p$ segment was established by DAPI banding (Fig. 1B), by $5^{\prime}$-bromo-2'-deoxyuridine (BrdU) banding, and by hybridization with a $1 \mathrm{p} 36.3$ probe (data not shown); the $1 \mathrm{p} 36.3$ probe additionally revealed deletion of this subregion in one of the apparently complete 1 chromosomes. The overall picture was of a nearly trisomic representation of chromosome 1 , with a common breakpoint, and subsequent translocation.

In TC 593, the glioblastoma line, an even more complex pattern of numerical and structural chromosome 1 aberrations was observed. In a sample of 50 metaphase spreads, the majority $(52 \%)$ showed six aberrant chromosomes that were decorated; $14 \%$ of the metaphases showed five aberrant chromosomes, and $34 \%$ showed higher numbers of chromosome 1 segments (up to 14). Figures $1 C$, D, and 4 show the most typical, predominant karyotype and demonstrate our rapid definition of chromosome 1 abnormalities in this cell line. Aberrations included three acrocentric chromosomes with a consistent breakpoint in $1 \mathrm{p} 1$, chromosomes with a deletion of the distal part of 1q, a submetacentric translocation chromosome with a loss of the complete 1q, and an iso(1p) marker chromosome (see Fig. 4).

Chromosome 4. In TC 620, chromosome 4-specific inserts decorated one apparently complete chromosome 4 , and three additional chromosomes with segments containing chromosome 4 DNA (Figs. 2F, 4). These latter segments on translocation chromosomes would have been difficult to rapidly and unambiguously define with banding procedures alone. The smallest of the translocated chromosome 4 segments formed part of an approximately metacentric chromosome. The two larger segments were found on submetacentric chromosomes of different overall size. In the smaller chromosome, the short arm and part of the long arm of 4 were present with an apparent breakpoint at $4 \mathrm{q} 2$, i.e. 4 pter-4q2. In the larger submeta- 

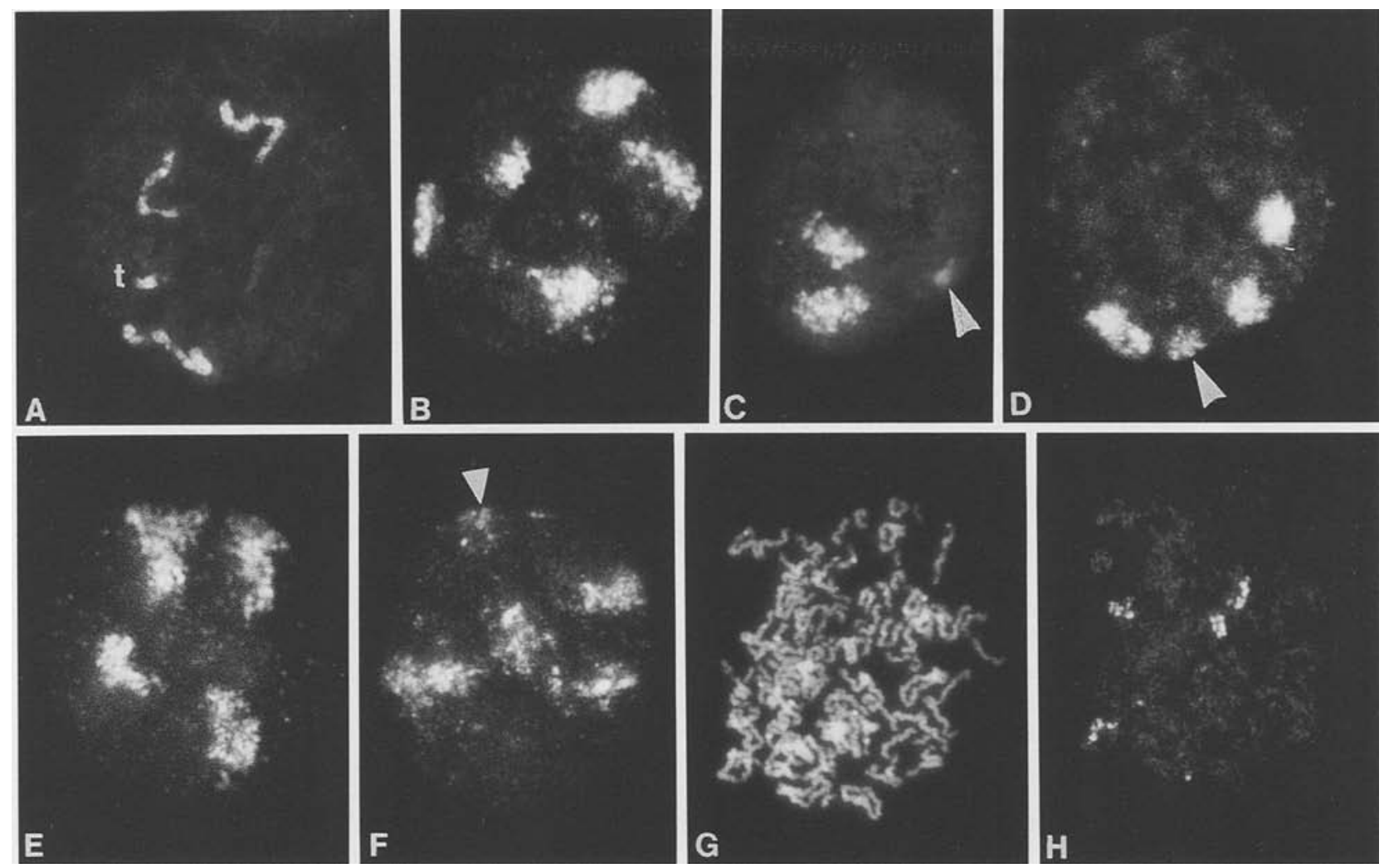

Fig. 5A-H. Representative prophase (A) and interphase nuclei (B-F) reflect metaphase abnormalities in glioma lines (cf. Figs. 1-4). A Detection of the $7 p$ translocation $(t)$ in a prophase TC 620 nucleus. $\times 1,000$. B Five well-separated chromosome 7 domains detected in TC 593. $\times 1,240$. C Two large and one very small 18 domains (indicated by arrow head) in TC $620 . \times 1,450$. D Four chromosome 18 domains in TC 593 ; one of these signals (arrow) appears smaller. $\times 1,450$. E Four chromosomal 1 domains in TC 620 (cf. Fig. 1 A, B). $\times 1,200$. F At least five chromosome 1 domains in TC 593; one (arrowhead) is appreciably smaller than the others. $\times 1,250$. G, H CISS hybridization of a technically poor metaphase spread of TC 593 poststained with DAPI $(\mathbf{G})$ still highlights four distinct chromosomes bearing 18 sequences $(\mathbf{H}) . \times 1,000$

centric chromosome, a region that may represent the rest of 4 (4q2-qter) appears. Thus the predominant karyotype of TC 620 showed only slightly more than two equivalents of chromosome 4 (see also the area measurements described below). The non- 4 regions have not been further defined.

In TC 593, there were generally only two chromosomes decorated by chromosome 4 DNA inserts, and both of these were compatible with normal 4 chromosomes. Approximately $30 \%$ of the metaphase spreads in TC 593 showed an additional submetacentric chromosome with chromosome 4 material (Fig. 2E). Thus, although both 4 chromosomes were apparently normal, there was a significant under-representation of this chromosome in this pseudotetraploid line (Fig. 4).

Chromosome 7. Three complete 7 chromosomes, and one smaller metacentric chromosome containing translocated 7 material were typically found in TC 620 metaphase spreads (Figs. 3A, 4). The translocated chromosome 7 material included the short arm of chromosome 7 (as shown by DAPI banding; cf. Fig. 3B) and the centromeric heterochromatin with the breakpoint in $7 \mathrm{q} 1$ (see also below).

In TC 593, five chromosomes entirely decorated by chromosome 7 insert probes were regularly observed (Figs. 2G, $3 E)$. Four of these appeared to represent complete number 7 chromosomes, whereas one was smaller and metacentric. DAPI banding (Fig. 3E, insert) and size measurements (cf. Fig. 9) were consistent with an iso(7p). This conclusion was further supported by double in situ hybridization experiments with biotinylated chromosome 7 inserts (detected with avidinFITC) and chromosome 7-specific alphoid AAF labeled sequences (detected with tetramethylrhodamine isothiocyanate (TRITC) conjugated second antibodies). They showed that only the four complete 7 chromosomes, contained a detectable 7 centromeric signal (metaphase, Fig. 2G, H; interphase, Fig. 2I, $\mathrm{J})$. Thus, the iso(7p) marker chromosome did not have a characteristic centromeric region as it lacked both the 7 alphoid sequences and a small block of heterochromatin at $7 \mathrm{q} 11$ (see Fig. 3E, insert). In contrast all four 7 chromosomes of TC 620 were labeled with the 7 alphoid probe (data not shown).

Chromosome 18. In TC 620 two apparently complete 18 chromosomes and a truncated minute chromosome were entirely decorated (Figs. 3C, D, 4). This truncated chromosome is $18 \mathrm{q}-$ (and possibly also $18 \mathrm{p}-$ ). The rest of the chromosome 18 region(s) was never detected.

Three translocation chromosomes involving chromosome 18 material were typically detected, in addition to an apparently normal chromosome 18 in TC 593 metaphase spreads (Figs. 3F, G, 4). In a minor proportion of metaphases there was a small additional translocation observed. The exact chromosomal region from which this translocated 18 material derived could not be resolved by DAPI staining. The predominant karyotype for 18 is therefore close to tetrasomic in this cell line, but is under-represented in the pseudotriploid TC 620 .

Both the 18q-marker chromosome in TC 620 and the three translocated 18 chromosomes in TC 593 hybridized strongly to 
a chromosome 18-specific alphoid repeat. Accordingly, both intact and aberrant 18 chromosomes could also be counted after in situ hybridization with this centromeric probe (Cremer et al. 1988; see also below). DAPI banding and hybridization to 18-specific alphoid repeats indicated that these translocation chromosomes include the entire 18q region and the centromere, with breakpoints in $18 \mathrm{p}$.

Chromosome 22. Two apparently normal 22 chromosomes were visualized in most TC 620 and TC 593 metaphase spreads (Fig. 6D). It was difficult to ascertain small translocations of this chromosome since hybridization with chromosome 22 inserts resulted in some cross-hybridization to other chromosomes. Some of this cross-hybridization is probably due to shared sequences from the nucleolus organizer regions (on five normal acrocentric human chromosomes) and to shared sequence motifs at the centromeres (McDermid et al. 1986; Lichter et al. 1988).

Finally, it should be noted that in contrast to conventional banding analysis, the current experimental approach clearly delineates numerical and structural chromosome aberrations in metaphase spreads of very poor quality (Fig. $5 \mathrm{G}, \mathrm{H}$ ) or in early prophase nuclei (Fig. 5A). These preparations are not accessible to banding analysis, as the chromosomes extensively overlie each other.

\section{Evaluation of chromosome domains in interphase nuclei}

One potential advantage of in situ methods is that individual human chromosomes may be directly visualized as discrete territories in interphase nuclei (Manuelidis 1985; Schardin et al. 1985; Pinkel et al. 1986) and thus can be of value in the analysis of solid tumor specimens. This feature of nuclear topography, also apparent in the malignant cells examined here (Figs. 2A-D, I, 5B-F), was evaluated for its accuracy and diagnostic usefulness. Figure $5 \mathrm{~A}$ shows three apparently complete 7 chromosomes and one translocated $7 \mathrm{p}$ arm in a prophase TC 620 nucleus. Figures 2I, and 5B show five chromosome 7 domains in interphase nuclei of TC 593, as previously depicted in metaphase spreads. Figure 5C shows a TC 620 interphase nucleus with two 18 domains of comparable sizes to those seen in normal diploid nuclei (see Lichter et al. 1988). A third, appreciably smaller, decorated 18 domain was also detected and represents the truncated 18 chromosome seen in metaphase spreads described above. Figure 5D shows four chromosome 18 domains in an interphase nucleus of TC 593, which again is comparable to the numbers in metaphase nuclei. Figure 5E shows a TC 620 interphase nucleus with four chromosome 1 domains, while Fig. $5 \mathrm{~F}$ shows a TC 593 nucleus with at least five separate chromosome 1 domains (compare Fig. 1A, B and C, D, respectively).

While the hybridization patterns of nuclei shown in Fig. 5 were highly characteristic for each cell line, counts of interphase chromosome domains have some inherent difficulties. As an example, Fig. 6A (dark columns) presents an analysis of the counts of labeled interphase domains in randomly selected nuclei of diploid human lymphocytes hybridized with 7 library inserts. Although the number and relative size of chromosome-specific domains can be accurately assessed in the majority of nuclei, not all nuclei present a reliable index of the chromosomal constitution, since a considerable fraction of nuclei reveal only one decorated domain and occasional nuclei show no signals. Furthermore all domains are not always clearly separable in these 2-D preparations.
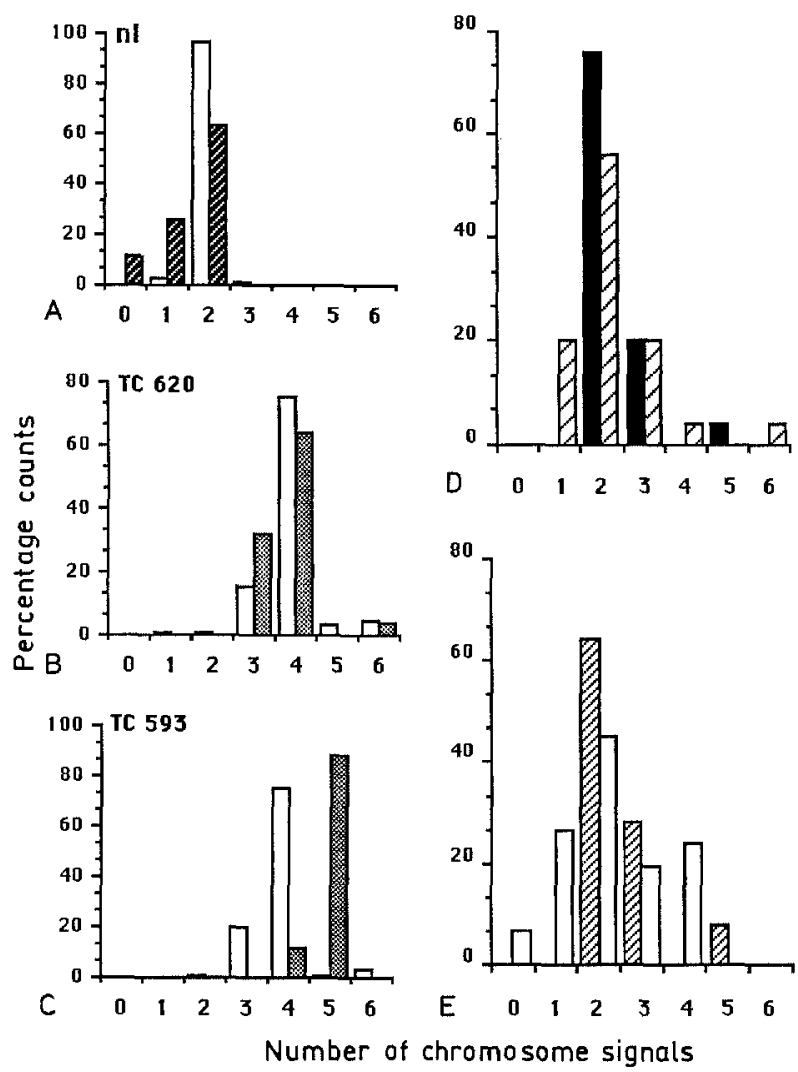

Fig. 6A-E. Interphase and/or metaphase counts of chromosomes 7 (A-C), 22 (D) and 4 (E). Interphase counts were performed on 150 nuclei of well-hybridized preparations. For metaphase counts $>25$ complete DAPI-stained spreads were evaluated. A-C Counts of 7 specific alphoid repeats (white columns) compared to 7 library inserts (shaded columns): $\mathbf{A}$ interphase nuclei of phytohemagglutinin-stimulated human lymphocytes $(46, X Y)$; B TC 620 interphase nuclei (7-specific alphoid repeat) and metaphase spreads (7 library inserts); C TC 593 interphase nuclei (7-specific alphoid repeat) and metaphase spreads (7 library inserts). High stringency hybridization (see Materials and methods) of 7 alphoid repeat was used to avoid cross-hybridization to other chromosomes. In cases of double hybridization with both 7 library inserts and alphoid repeat (shown in Fig. 2 G-I) standard conditions with $50 \%$ formamide were sufficient to avoid crosshybridization, possibly due to the presence of human competitor DNA. D Counts of chromosome 22 (library inserts) in metaphase spreads of TC 620 (black columns) and TC 593 (shaded columns). For comparison, CISS hybridization was simultaneously performed with 7 library inserts in these experiments as an internal control (see B, C and Fig. 7). E Interphase counts (white columns) and metaphase counts (shaded columns) compared in TC 593 hybridized with chromosome 4 inserts. Note the ratios of cells with 2 vs 3 signals are the same in metaphase and interphase

Figure 6 shows representative counts of these preparations. In agreement with TC 593 metaphase counts of chromosome 4 , nuclear counts generally showed two clearly separated domains (Fig. 6E). However, the percentage of two-signal preparations was smaller in interphase than in metaphase ( $45.3 \%$ vs. $64 \%)$. This artifactual decrease was largely due to a corresponding increased percentage of nuclei showing only one decorated domain or no signal at all. Counts of zero or one chromosome 4 were not present in metaphase spreads. Significantly, $19.3 \%$ of the interphase TC 593 nuclei displayed three clearly separated chromosome 4 domains, and these extra domains were not present in interphase nuclei of diploid human lymphocytes hybridized to this or other libraries under the 


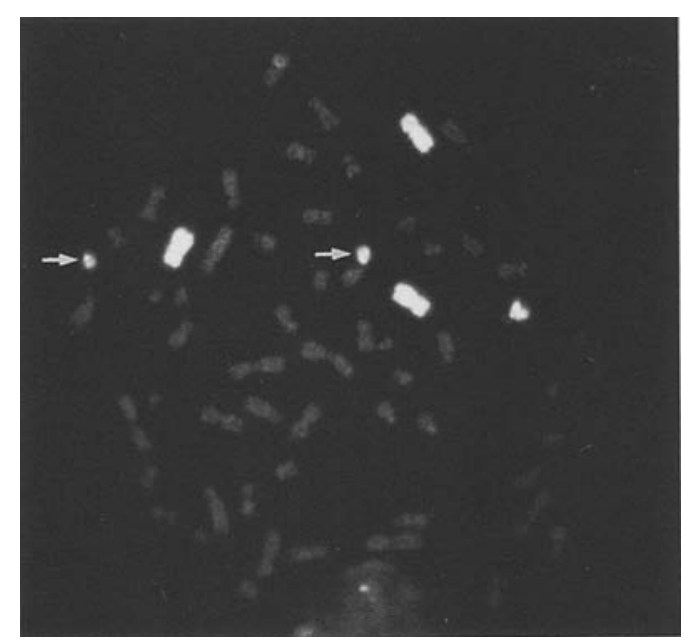

Fig. 7. TC 620 metaphase spread after double hybridization with inserts from both 7 and 22 chromosomes (both labeled with biotin and detected with avidin-FITC). Two strongly decorated 22 chromosomes (arrows), three complete 7 chromosomes, and the metacentric marker chromosome containing 7 pter-q11 are seen

same conditions (Fig.6A; Lichter et al. 1988). Finally, the ratio of two versus three domains was identical for both metaphase and interphase cells. Thus interphase nuclei can be reliably used for the detection of extra copies of a single chromosome or chromosomal segment but have limited reliability for detecting the loss of chromosome copies.

In situ hybridization of probes from subregions of interphase chromosomes may more accurately reflect general counts of chromosomal constitution than library probes (Fig. 6A), provided they are done under appropriately high stringency conditions (Rappold et al. 1984; Cremer et al. 1986, 1988). However, such regional segment probes do not delineate translocated elements or aberrant chromosomes that lack this segment. Therefore such probes are also not entirely accurate. For example, counts of chromosome 1 in TC 620 and TC 593 with a probe specific for 1q12 (Cremer et al. 1988) indicated fewer 1 chromosomes than shown here with CISS hybridization (Fig. 4). Counts of chromosome 7 using only a centromeric sequence further emphasize this point (see above). Double in situ hybridization with the AAF-modified 7 alphoid probe and biotinylated chromosome 7 library inserts typically showed interphase nuclei with five domains, of which only four were simultaneously labeled by the centromeric probe (Figs. 2I, J, $6 \mathrm{C})$. In TC 620 , however, both probes gave identical results (Fig. 6B).

\section{Over-representation and under-representation of specific chromosomes}

We assessed relative chromosomal dosage in these glioma lines, and were especially interested in chromosome 7 , which has been noted to be generally over-represented in gliomas (Bigner et al. 1986; Shapiro 1986). For comparison, other individual chromosome probes were used as controls. Metaphase chromosome counts have shown that TC 620 is pseudotriploid with a modal number of 64 chromosomes, while TC 593 is pseudotetraploid with a modal number of 83 (Manuelidis and Manuelidis 1979). Accordingly, a chromosome and its segments together would be present in a balanced state if three complete copies were present in TC 620, and four in TC 593.

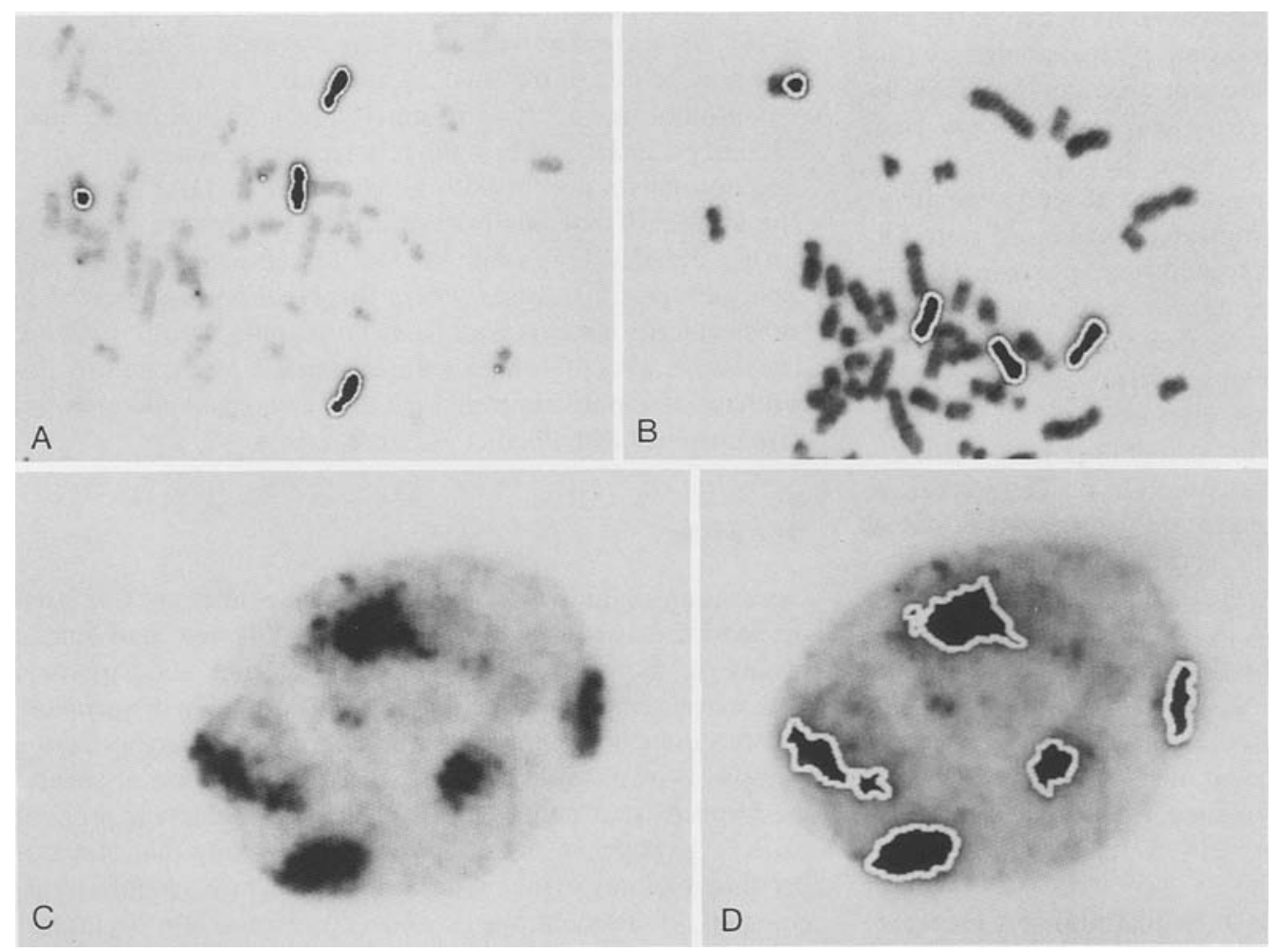

Fig. 8A-D. Digitized images of metaphase spreads and interphase nuclei from malignant glioma cells hybridized to chromosome 7 inserts. A, B Two TC 620 metaphase spreads analyzed under standard conditions (A) and under less optimal conditions (B). Note that three complete 7 chromosomes and the translocated $7 \mathrm{p}$ arm can be discriminated by the computer (outlined areas) in $\mathbf{B}$ despite a high background of other chromosomes. C, D Digitized TC 593 interphase nucleus (shown photographically in Fig. 5B), automatically outlined (D) for area determinations 
Table 1. Mean number of chromosome equivalents measured by digital image analysis in malignant glioma cell lines after CISS hybridization. Twenty-four metaphase spreads showing the predominant number of chromosomes decorated with DNA inserts from libraries of chromosomes 4,7 and 18 were compared to twenty-eight interphase nuclei with well-separated domains using the same probes. Images were taken under identical (linear film) conditions and digitized. In each metaphase spread, areas obtained for each normal and aberrant chromosome were divided by the mean area obtained for $n$ apparently complete chromosomes. In interphase nuclei, domains were compared assuming that the $n$ largest labeled domains represented complete (normal) interphase chromosomes. Thus the sum of these normalized values represents a measure of the number of specific chromosome equivalents in a single cell. The mean values of several cells are shown for each case. The mean numbers of chromosome equivalents obtained for interphase and metaphase cells show a strong overall correlation coefficient of $r=+0.95$. Compared with area measurements, the mean numbers of chromosome equivalents determined by 2 -D intensity integrals (see Materials and methods; data not shown) showed an overall correlation coefficient of $r=+0.99$

\begin{tabular}{lllll}
\hline \multirow{2}{*}{$\begin{array}{l}\text { Chromo- } \\
\text { some }\end{array}$} & Cell line & \multicolumn{3}{l}{ Chromosome equivalents } \\
\cline { 3 - 5 } & & Interphase & Metaphase & Expected \\
\hline \multirow{2}{*}{4} & TC593 & 2.0 & 2.0 & 4.0 \\
& TC620 & 2.5 & 2.4 & 3.0 \\
7 & TC593 & 4.4 & 4.6 & 4.0 \\
& TC620 & 3.5 & 3.3 & 3.0 \\
& TC593 & 3.0 & 3.6 & 4.0 \\
& TC620 & 2.5 & 2.3 & 3.0 \\
\hline
\end{tabular}

A relative over-representation is present if more than these respective copy numbers can be demonstrated. A number lower than the expected (trisomic or tetrasomic) value indicates that the chromosome is relatively under-represented in the karyotype. In cases where additional DAPI banding information was sufficient to define the selectively decorated abnormal chromosome, the chromosome pieces labeled by the chromosome-specific inserts were put together for analysis (Fig. 4). In the second approach, computer analyses were used to independently verify these results (see below).

TC 620 analyzed by banding showed the equivalent of three 1 chromosomes and thus indicated a balanced state for this chromosome. The same was true for the $1 \mathrm{p}$ arm in TC 593 which was present in four copies. However, the distal part of $1 \mathrm{q}$ was under-represented in TC 593 (see the detailed description given above). In both glioma lines 7q appeared to be balanced, while $7 \mathrm{p}$ was over-represented once in TC 620 and twice in TC 593. Additionally, in both glioma lines chromosome 22 was clearly under-represented. In order to confirm this finding, double in situ hybridization with inserts of chromosomes 7 and 22 was performed. An example of this is shown in Fig. 7 and demonstrates over-representation of 7 DNA and under-representation of $22 \mathrm{DNA}$ in the same cell. Metaphase counts done in both cell lines by this method of analysis are depicted for chromosome 7 in Fig. 6B, C (dark columns) and for chromosome 22 in Fig. 6D. In summary, these two gliomas both show relative under-representation of chromosome 22 and over-representation of the $7 \mathrm{p}$ arm. The significant underrepresentation of chromosome 4 in TC 593, and a portion of 4 in TC 620 is also notable.

Digitized images were also used to quantitatively measure decorated areas in metaphase preparations and in interphase cells where chromosomal domains were well resolved. Examples are shown in Fig. 8. Quantitative evaluation of chromosome equivalents (Table 1) indicated highly concordant num-

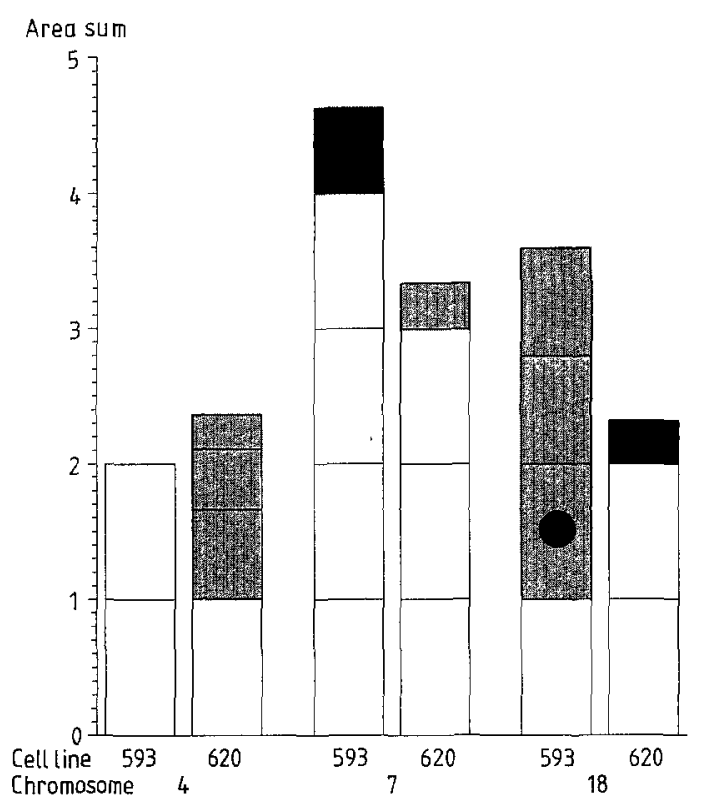

Fig. 9. Relative size of decorated normal and aberrant chromosomes 4,7 and 18 in typical metaphase spreads $(n=24)$ from glioma cell lines TC 593 and TC 620 . Individual areas were normalized so that a complete chromosome is represented by an area of 1 (see legend to Table 1). The total added signals reflect the number of specific chromosome equivalents present. The white regions correspond to apparently normal chromosomes, the black regions indicate small free chromosome segments entirely decorated by specific library inserts, and translocated segments are shaded. One of the three translocated 18 chromosomes in TC 593 represents a complete chromosome by this measurement (indicated by the black dot), while the two other translocations are slightly smaller, possibly due to the small sample size

bers for interphase versus metaphase in 5 of 6 examples; only in TC 593 decorated with 18 inserts was there a discrepancy. This may be due to the small sample size.

Chromosome equivalents derived from digital image analysis independently confirm the relative representation of target chromosomes noted in both glioma lines by DAPI banding. The segments that comprise the total metaphase signal are further detailed graphically in Fig. 9. Computer analysis was especially useful in cases where the breakpoints involved in translocated segments could not be unambiguously defined. They were also of value in a quantitative assessment of interphase-metaphase correlations, and of normal and aberrant chromosomes with distinctly different sizes.

\section{Discussion}

Decoration of individual human chromosomes by CISS hybridization with DNA libraries provides a new and simple means for the rapid detection of chromosome aberrations in both mitotic and interphase tumor cells. Formation of aneuploid chromosomal complements due to segregational errors during mitosis (Boveri 1914) has been considered as one of several mechanisms that might lead to cells with malignant propensities (e.g. Harris 1986; Shapiro 1986). Factors that may not produce obvious cytogenetic changes, such as chemical carcinogens, mutational agents and viruses may also be important in neoplastic initiation and expression. Ultimately, however, detectable chromosomal changes are often apparent (Mitelman 1985). In this setting it is pertinent to be able to evaluate individual tumor cells of a population. 
We have documented structural and quantitative changes in two human glioma lines, including the loss and gain of entire individual chromosomes, and of chromosomal subregions, and have been able to characterize both minor and predominant karyotypic features in each cell line. All chromosomes tested so far, namely $1,4,7,18$ and 22 , clearly highlighted numerical and/or structural aberrations, some of which were subtle. The biological implications of our findings suggest that this new approach may be of general value in tumor cytogenetics.

In agreement with previous reports (Shapiro 1986; Bigner et al. 1986, 1987; Rey et al. 1987) we have found an over-representation of chromosome 7 and an under-representation of chromosome 22 in these two glioma lines. These characteristics are still apparent after long-term propagation in culture ( $>20$ years). It is of interest to note that in both cell lines $7 \mathrm{p}$ but not $7 \mathrm{q}$ was over-represented. The $c-e r b B$ oncogene (EGFR) has been mapped to 7p12-14 (Merlino et al. 1985). It can be over-expressed in human glioblastoma cell lines with polysomy of chromosome 7 (Henn et al. 1986), and amplification of $c$-erbB has often been found in malignant gliomas (Liberman et al. 1985; Yamazaki et al. 1988; Bigner et al. 1988). This oncogene does not show amplification on Southern blot analysis in either TC 620 or TC 593, and in TC 593 there are no rearrangements, insertions or deletions involving $c-e r b B$ (T. Chen and L.Manuelidis, unpublished data). Our finding of over-representation of $7 \mathrm{p}$, together with others (Bigner et al. 1987; Rey et al. 1987) indicates, however, that the 7p13q11 region which also contains $A$-raf 2 (Bloomfield et al. 1987) is specifically involved in most malignant human gliomas.

Several groups have reported that glioma lines secrete platelet-derived growth factor or a PDGF-like molecule (reviewed by Shapiro 1986) that is coded for by the $c$-sis oncogene (PDGFB) located on 22q12.3-13.1. There is an apparent discrepancy between the overexpression of PDGF and the under-representation of chromosome 22. It has been suggested that a chromosome 22-translocation involving $c$-sis might remove constraints that govern the normal expression of this gene (Shapiro 1986). We have not been able to demonstrate a small. 22-translocation in TC 620 or TC 593 but this may be due to technical limitations (see the Results). The possibility should also be considered that chromosome 22 contains an as yet unidentified recessive gene(s) (Knudson 1986; Hansen and Cavanee 1987) predisposing to gliomas when absent or underexpressed.

The possible contribution, if any, of numerical and/or structural aberrations of chromosomes 1, 4 and 18 is not well understood at present (cf. Bloomfield et al. 1987; Al-Saadi et al. 1987). Chromosome 1 aberrations are involved in many solid tumors (Atkin 1986) and in neuroectodermal neoplasms (Brodeur et al. 1981; Bigner et al. 1986). Thus the aberrations of chromosome 1 detected in this stucy are not surprising. However, in both glioma lines, breakpoints close to or within $1 \mathrm{p} 1$ were observed, and this deserves further analysis of oncogenes such as $N$-ras, as well as other genes in this region. $N$ ras is not amplified or rearranged in TC 593 or TC 620 (T.Chen and L.Manuelidis, unpublished data), although point mutations in this oncogene which can be significant, have not been determined in these lines. The under-representation of chromosome 4 suggests that further analysis of this chromosome in gliomas is warranted.

The present studies demonstrate the utility of this method for the rapid assessment of breakpoints in tumor cells, such as the $1 \mathrm{p} 1$ breakpoint noted above. Chromosome segments consistently involved in the development or progression of a given type of tumor may also be rapidly identified (e.g. 7p13-q11). In some cases the delineation of specific chromosomal segments or of specific breakpoints may suggest a role for genes already known to be located in the defined area. The current strategy is also capable of revealing previously unidentified specific chromosome segments that can directly lead to new and pertinent molecular analyses.

A more precise definition of breakpoints and of the chromosomal regions involved in deletion, translocation, duplication or amplification can be achieved using either single copy probes or regional-specific repititive probes. Suppression hybridization can also be combined with "classic" chromosome banding procedures, e.g. DAPI banding (this paper) or replication banding (Manuelidis and Borden 1988). Furthermore, the double in situ hybridization described in this paper makes it possible to simultaneously decorate both complete individual chromosomes and chromosomal subregions.

We have demonstrated that individual chromosomes can be easily distinguished and quantitatively measured in very complex karyotypes, and reliably assessed in non-optimal metaphase spreads and early prophase nuclei where many overlaps with other chromosomes are typical. Interpretation of banding patterns in specifically decorated chromosome segments is greatly facilitated by the fact that the chromosomal assignment is unambiguous, and cannot be confused with partial chromosomes or translocated segments from other chromosomes that contain a similar or even identical banding pattern. Thus, further banding analysis can simply focus on the individual chromosome or segment in question. Library or regional probes can also be applied to tissue sections and solid tumors (Manuelidis and Borden 1988; P.Emmerich, H. Walt and T.Cremer, unpublished data). Thus one can potentially delineate specific chromosomal changes that are characteristic of a more malignant phenotype in a heterogeneous tumor cell population. Quantitative measurements of the sizes of interphase domains, as shown here, provide an additional approach to analyze the genetic complement of tumor cells.

With further developments of multi-probe hybridization and detection protocols to increase the spectrum of chromosomal targets that can be simultaneously visualized, the utility of the current approach will be further enhanced for tumor cytogenetics. In addition it suggests a novel way to band individual mitotic and interphase chromosomes. While the practicability of such an approach needs to be experimentally developed, suppression hybridization techniques even in their present state, provide a unique tool to rapidly screen tumor cell populations for the presence and DNA constitution of specific marker chromosomes.

Acknowledgements. The skillful technical assistance of Ed Zelazny for tissue culture, of Chieh-ju Chang Tang for library DNA preparations and of Chris Labanowski for photographic work is gratefully acknowledged. T.C. is the recipient of a Heisenberg stipendium and P.L. of a training grant, both from the Deutsche Forschungsgemeinschaft. This work was supported by NIH grant CA-15044 (to L.M.) and GM-32156 and GM-40115 (to D.C.W.).

\section{References}

Al-Saadi A, Latimer F, Madercic M, Robbins T (1987) Cytogenetic studies of human brain tumors and their clinical significance. II. Meningioma. Cancer Genet Cytogenet 26:127-141 
Atkin NB (1986) Chromosome 1 aberrations in cancer. Cancer Genet Cytogenet 21:279-285

Bigner SH, Mark J, Bullard DE, Mahaley MS Jr, Bigner DD (1986) Chromosomal evolution in malignant human gliomas starts with specific and usually numerical deviations. Cancer Genet Cytogenet 22:121-135

Bigner SH, Wong AJ, Mark J, Mublbaier LH, Kinzler KW, Vogelstein B, Bigner DD (1987) Relationship between gene amplification and chromosomal deviations in malignant gliomas. Cancer Genet Cytogenet 29:165-170

Bigner SH, Burger PC, Wong AJ, Werner MH, Hamilton SR, Mublbaier LH, Vogelstein B, Bigner DD (1988) Gene amplification in malignant human gliomas: clinical and histopathologic aspects. J Neuropathol Exp Neurol 47:191-205

Bloomfield CD, Trent JM, Berghe H van den (1987) Report of the committee on structural chromosome changes in neoplasia. (9th International Workshop on Human Gene Mapping) Cytogenet Cell Genet 46:344-366

Boveri T (1914) Zur Frage der Entstehung maligner Tumoren. Fischer, Jena

Brodeur GM, Green AA, Hayes FA, Williams KJ, Tsiafis HA (1981) Cytogenetic features of human neuroblastomas and cell lines. Cancer Res $41: 4678-4686$

Burns J, Chan VTW, Jonasson JH, Fleming KA, Taylor S, McGee J O'D (1985) Sensitive system for visualizing biotinylated DNA probes hybridized in situ: rapid sex determination of intact cells. J Clin Pathol 38:1085-1092

Cremer T, Landegent JE, Brückner $\mathrm{H}$, Scholl HP, Schardin M, Hager HD, Devilee P, Pearson PL, Ploeg M van der (1986) Detection of chromosome aberrations in the human interphase nucleus by visualization of specific target DNAs with radioactive and non-radioactive in situ hybridization techniques: diagnosis of trisomy 18 with probe L1.84. Hum Genet 74:346-352

Cremer T, Tesin D, Hopman AHN, Manuelidis L (1988) Rapid interphase and metaphase assessment of specific chromosomal changes in neuroectodermal tumor cells by in situ hybridization with chemically modified DNA probes. Exp Cell Res 176:199-220

Hansen MF, Cavenee WK (1987) Genetics of cancer predisposition. Cancer Res 47:5518-5527

Harris H (1986) The genetic analysis of malignancy. J Cell Sci [Suppl] $4: 431-444$

Henn W, Blin N, Zang KD (1986) Polysomy of chromosome 7 is correlated with overexpression of the erbB oncogene in human glioblastoma cell lines. Hum Genet 74:104-106

Knudson AG (1986) Genetics of human cancer. Annu Rev Genet 20: 231-251

Landegent JE, Jansen in de Wal N, Baan R, Hoeijmakers JHJ, Ploeg $M$ van der (1984) 2-Acetylaminofluorene-modified probes for the indirect hybridocytochemical detection of specific nucleic acid sequences. Exp Cell Res 153:61-72

Larizza L, Schirrmacher V (1984) Somatic cell fusion as a source of genetic rearrangement leading to metastatic variants. Cancer $\mathrm{Me}$ tastasis Rev 3:193-222

Libermann TA, Nusbaum HR, Razon N, Kris R, Lax I, Soreq H, Whittle N, Waterfield MD, Ullrich A, Schlessinger J (1985) Amplification, enhanced expression and possible rearrangement of EFG receptor gene in primary human brain tumors of glial origin. Nature $313: 144-147$
Lichter P, Cremer T, Borden J, Manuelidis L, Ward DC (1988) Delineation of individual human chromosomes in metaphase and interphase cells by in situ suppression hybridization using recombinant DNA libraries. Hum Genet 80:224-234

Manuelidis L (1985) Individual interphase domains revealed by in situ hybridization. Hum Genet 71:288-293

Manuelidis L, Borden J (1988) Reproducible compartmentalization of individual chromosome domains in human CNS cells revealed by in situ hybridization and three dimensional reconstruction. Chromosoma 96:397-410

Manuelidis L, Manuelidis EE (1979) Surface growth characteristics of defined normal and neoplastic neuroectodermal cells in vitro. In: Zimmermann HM (ed) Progress in neuropathology, vol 4. Raven Press, New York, pp 235-266

McDermid HE, Duncan AMV, Higgins MJ, Hamerton JL, Rector E, Brasch KR, White BN (1986) Isolation and characterization of an alpha-satellite repeated sequence from human chromosome 22. Chromosoma 94:228-234

Merlino GT, Ishii S, Whang-Peng J, Knutsen T, Xu Y-H, Clark AJL, Stratton RH, Wilson RK, Ma DP, Roe BA, Hunts JH, Shimizu N, Pastan I (1985) Structure and localization of genes encoding aberrant and normal epidermal growth factor receptor RNAs from A431 human carcinoma cells. Mol Cell Biol 5:1722-1734

Mitelman F (1985) Catalogue of chromosome aberrations in cancer, 2nd edn. Liss, New York

Pinkel D, Straume T, Gray JW (1986) Cytogenetic analysis using quantitative, high sensitive, fluorescence hybridization. Proc Natl Acad Sci USA 83:2934-2938

Rappold G, Cremer T, Hager HD, Davies KE, Müller CR, Yang T (1984) Sex chromosome positions in human interphase nuclei as studied by in situ hybridization with chromosome specific DNA probes. Hum Genet $67: 317-325$

Rey JA, Bello JM, Campos JM de, Kusak ME, Moreno S (1987) On trisomy of chromosome 7 in human gliomas. Cancer Genet Cytogenet $29: 323-326$

Schardin M, Cremer T, Hager HD, Lang M (1985) Specific staining of human chromosomes in Chinese hamster $\times$ man hybrid cell lines demonstrates interphase chromosome territories. Hum Genet 71 : 281-287

Shapiro JR (1986) Biology of gliomas: heterogeneity, oncogenes growth factors. Semin Oncol 13:4-15

Showe LC, Croce CM (1987) The role of chromosomal translocations in B- and T-cell neoplasia. Annu Rev Immunol 5:253-277

Trask B, Engh G van den, Pinkel D, Mullikin J, Waldman F, Dekken H van, Gray J (1988) Fluorescence in situ hybridization to interphase cell nuclei in suspension allows flow cytometric analysis of chromosome content and microscopic analysis of nuclear organization. Hum Genet 78:251-259

Waye JS, England SB, Willard HF (1987) Genomic organization of chromosome-specific alpha satellite DNA on human chromosome 7: evidence for two distinct alphoid domains on a single chromosome. Mol Cell Biol 7:349-356

Yamazaki H, Fukui Y, Ueyama Y, Tamaoki N, Kawamoto T, Taniguchi S, Shibuya M (1988) Amplification of the structurally and functionally altered epidermal growth factor receptor gene (cerbB) in human brain tumors. Mol Cell Biol 8:1816-1820

Received June 27, 1988 / Revised July 15, 1988 